\title{
In vitro resistance to daptomycin of staphylococcus aureus and enterococcus spp. strains
}

\author{
${ }^{1}$ Emergency County Clinical Hospital "Sf. Apostol Andrei" Constanţa \\ ${ }^{2}$ Faculty of Medicine, University „Ovidius” of Constanţa
}

\begin{abstract}
Daptomycin is the first antibiotic in a new class of cyclic lipopeptides, active in vitro against Gram - positive cocci. The mechanism of action is a rapid depolarization of the bacterial membrane potential, loss of cytoplasmic contents, mainly $\mathrm{K}+$ ions and inhibition of protein, DNA and RNA synthesis, followed by the bacterial cell death. There were investigated 112 strains of Gram-positive cocci recommended for daptomycin susceptibility testing. Since the main clinical indications of daptomycin are the severe skin and soft tissue infections caused by methicillin-resistant Staphylococcus aureus, vancomicin-resistant enterococci and penicillinresistant Streptococcus spp, we tested daptomycin activity only in these species.

The susceptibility tests was performed by KirbyBauer disc-diffusion method on Muller-Hinton agar supplemented with $\mathrm{Ca} 50 \mathrm{mg} / \mathrm{ml}$. (CAMH - Ca 2+) as recommended by CLSI.

During the period in which we conducted the study we isolated 112 strains of Gram-positive cocci, which we tested sensitivity to daptomycin. Resistance to Daptomycin of Staphylococcus aureus (4.11\%) was
\end{abstract}

\section{Stan Irina}

Bd. Mamaia 334, Bl. ST6, ap.14

stan_irina1986@yahoo.com lower than resistance to vancomycin $(6.76 \%)$, but higher than the resistance to teicoplanin $(2.71 \%)$ and linezolid $(1.38 \%)$. In our study we did not identify daptomycin resistant strains of Enterococcus spp.

In severe infections that require more aggressive treatment using active antibiotics on MRSA, daptomycin is a valid therapeutic option, but a susceptibility test is required to ensure effective indication. There were no daptomycin resistant Enterococcus spp. strains

Keywords: Daptomycin, resistance, Staphylococcus, Enterococcus

\section{Introduction}

Daptomycin is the first antibiotic in a new class of cyclic lipopeptides, active in vitro against aerobic, facultative anaerobic Gram-positive Gram - positive cocci, including methicillin-resistant Staphylococcus aureus (MRSA), glycopeptyde-intermediate Staphylococcus aureus ( GISA), Penicillin- resistant Streptococcus spp and vancomycin-resistant enterococci (VRE).

Daptomycin is bactericidal. The mechanism of action is a rapid depolarization of the membrane potential, in the presence of $\mathrm{Ca}++$ ions, resulting in the formation of pores, with loss of cytoplasmic contents, mainly $\mathrm{K}+$ ions (Figure 1) and inhibition of protein, DNA and RNA synthesis, with bacterial 
death [1].

Daptomycin is indicated for treatment of complicated skin and soft tissue infections, endocarditis and bacteremia due to Gram posivie cocci. Although it is active in vitro on Streptococcus pneumoniae, Daptomycin is less efficient in the treatment of pneumonia, possible due to its inactivation by the pulmonary surfactant $[2,3]$.

Mechanisms of resistance to daptomycin are not well described. Genetic changes in the mprF gene (regulating cell membrane charge) has been correlated to daptomycin resistance. In vitro emergence of bacterial resistance most commonly occurs by spontaneous and chemical mutations, and by serial passage [10].

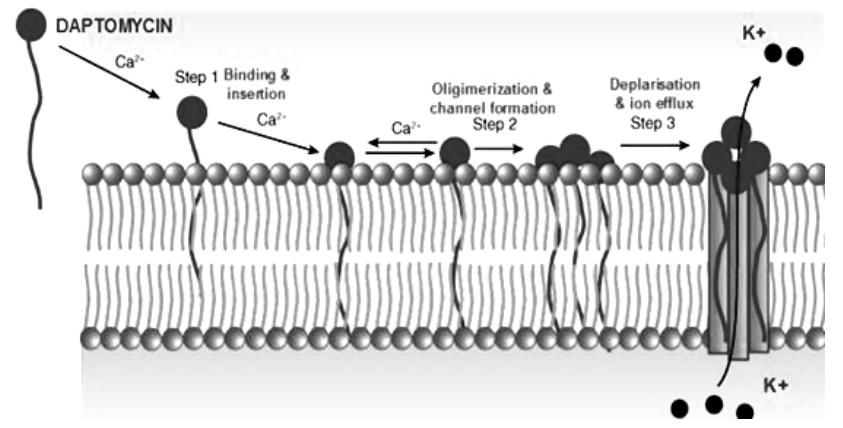

Figure 1 - Daptomycin mechanism of action (source: http://www.antibiotics-info.org)

\section{Objectives}

Were investigated 112 strains of Gram-positive cocci recommended for testing to daptomycin. Since the main clinical indications of daptomycin are the severe infections of the skin and soft tissue infections caused by methicillin resistant Staphylococcus spp, vancomycine - resistant enterococci and Penicillinresistant Streptococcus spp [4, 5], we tested daptomycin only in these species.

\section{Material and Method}

Were investigated various pathological products collected from outpatients. Specimens were secretions of skin lesions: pustules, acne lesions, eczema overgrowth, vaginal discharge, urethral discharge, urine cultures, sperm cultures. Samples were collected with sterile swabs and introduced in Amies conservation and transport medium tubes. Processing was carried out as quickly as possible after sampling. The study period was from January 2012 to July 2012.

Cultures from the pathological products were done on blood - agar medium plates, and BTBLA or CLED agar, depending on the pathological product. Incubation of the cultures was in usual aerobic environment, 24 hours at $370 \mathrm{C}$, with extension to 48 hours if at the first reading growth was not observed or culture did not look convincing.

Species identification was made on the culture appearance and biochemical characters.

Daptomycin susceptibility testing. Antibiograms were performed by the classical method (DST: Kirby - Bauer disc diffusion method). The culture medium used was Muller - Hinton agar without inhibiting substances, $\mathrm{pH}$ 7.2-7.4. An exception was the antibiogram for streptococci, for which Muller Hinton agar with blood was used. To test strains of Staphylococcus aureus to daptomycin, we used Mueller-Hinton using solid medium supplemented with Ca $50 \mathrm{mg} / \mathrm{ml}$ (CAMH- Ca2 + -supplemented) CLSI standard; 2010 [1,6].

Bacterial inoculum was represented by a suspension with a density of $0.5 \mathrm{Mc}$ Farland (about 108 microorganisms $/ \mathrm{ml}$ ), pure culture from the strain to be tested. Incubation was at $35^{\circ} \mathrm{C}$ (higher temperature testing can not detect MRSA strains) for 16-18h. Proposed breakpoints for daptomycin, when tested by disc diffusion method on Mueller Hinton agar medium with $50 \mathrm{mg} / \mathrm{ml} \mathrm{Ca}++$ correlate satisfactorily with MIC of $1 \mathrm{mg} / \mathrm{L}$, Muller Hinton broth determined supplemented with $50 \mathrm{mg} / \mathrm{ml} \mathrm{Ca}++$. The interpretation is as follows: inhibition zone diameter $>16 \mathrm{~mm}$ - Sensitive to daptomycin, inhibition zone 
diameter $<14 \mathrm{~mm}$ - resistant to daptomycin (Table I).

Table I - Interpretation table for Daptomycin susceptibility test

\begin{tabular}{|c|c|}
\hline Antibiotic & DAPTOMYCINE \\
\hline $\begin{array}{c}\text { MIC breakpoint }(\mathrm{mg} / \mathrm{L}) \\
\mathrm{S} \leq \mathrm{R}>\end{array}$ & 11 \\
\hline Disc content $(\mu \mathrm{g} /$ disc $)$ & $30 \mu \mathrm{g}$ \\
\hline $\begin{array}{c}\text { Zone diameter breakpoints } \\
\mathrm{S} \geq \mathrm{R}<\end{array}$ & $16 \mathrm{~mm} 14 \mathrm{~mm}$ \\
\hline Observations & \\
\hline
\end{tabular}

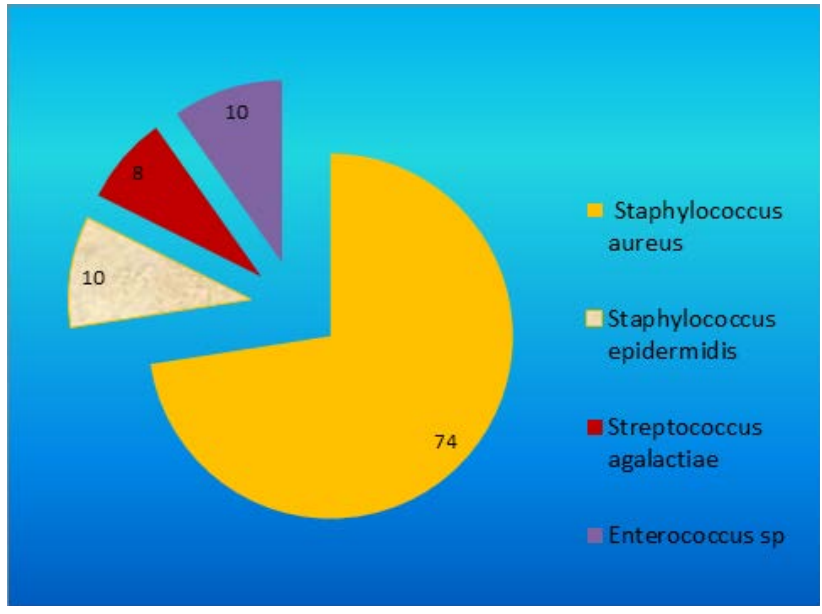

Figure 3 - Isolated bacterial species, tested to daptomycin

\section{Results and Discussion}

During the study we conducted in various pathological products from ambulatory patients, we isolated 112 strains of Gram-positive cocci recommended for testing daptomycin (Figure 2).

- Staphylococcus aureus - 74 strains

- Staphyloccus epidermidis - 10 strains

- Streptococcus agalactiae (group streptoccoci) - 8 strains

- Enterococcus sp. - 10 strains

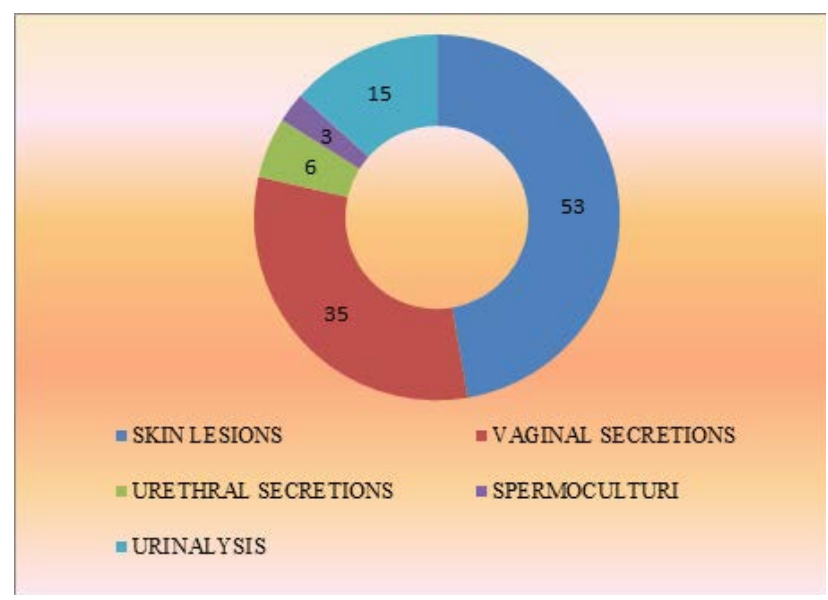

Figure 2 - Pathological specimens cultured

Since the main clinical indications of daptomycin are the severe infections of the skin and soft tissue infections caused by Staphylococcus spp. methicillin-resistant and vancomycin-resistant or, vancomycin-resistant enterococci and Streptococcus spp., Penicillin- resistant Daptomycin we tested only in these species. It is also indicated against linezolid -resistant bacteria. We compared the sensitivity of isolates strains to daptomycin, with susceptibility to linezolid.

The specimens were: skin lesions: pustules acne lesions, eczema overgrowth, vaginal discharge, urethral discharge, sperm cultures, urine cultures.

The results of the susceptibility tests, as summarised in Table 2, show that susceptibility to daptomycin (95.89\%) of Staphylococcus aureus was higher than susceptibility to vancomycin (93.24\%), but lower then sensitivity to teichoplanin (97.29\%).

Linezolid is highly active against Grampositive bacteria, including staphylococci, methicillin-resistant $\mathrm{S}$. aureus both (spectrum includes multiresistant strains) and S. aureus methicillinsensitive [7]. However, the treatment with linezolid is more expensive compared to daptomycin.

We have not identified any Streptococcus agalactiae, Enterococcus and Staphylococcus epidermidis strains to be resistant to daptomycin.

The 5 strains of vancomycin-resistant $\mathrm{S}$. aureus were susceptible to daptomycin.

We have found the presence of daptomycin 
resistance (with the zones of inhibition $0 \mathrm{~mm}$ ) in three strains of Staphylococcus aureus.

In all three cases presented, the strains resistant to Daptomycin were susceptible to Vancomycin. Moreover, we estimate that there were strains with multiple resistance: resistant to oxacillin (MRSA), macrolides, cephalosporins 2 nd and 3rd generations.

Table 2 - results of susceptibility tests for isolated $\mathrm{S}$. aureus strains

\begin{tabular}{|l|c|c|}
\hline Antibiotics & $\begin{array}{c}\text { No. of susceptible } \\
\text { strains }\end{array}$ & Percentage \\
\hline Penicilline (P) & 13 & $17.56 \%$ \\
\hline Ampicilline (AM) & 32 & $43.24 \%$ \\
\hline Lincomycine (LI) & 55 & $74.32 \%$ \\
\hline Tetracycline(TE) & 26 & $35.13 \%$ \\
\hline Erithromycine (E) & 47 & $63.51 \%$ \\
\hline Rifampicine (RIF) & 58 & $78.37 \%$ \\
\hline Norfloxacine (NOR) & 37 & $50 \%$ \\
\hline Oxacilline (OX) & 40 & $67.56 \%$ \\
\hline Gentamycine (GE) & 66 & $89.18 \%$ \\
\hline Co-trimoxazole (SXT) & 45 & $60.81 \%$ \\
\hline Cloramphenicol (C) & 11 & $14.86 \%$ \\
\hline Teichoplanine (TE) & 72 & $97.29 \%$ \\
\hline Vancomycine (VA) & 69 & $93.24 \%$ \\
\hline Linezolid (LNZ) & 73 & $98.62 \%$ \\
\hline Daptomycin (DAP) & 71 & $95.89 \%$ \\
\hline
\end{tabular}

\section{Clinical cases}

\section{Case 1}

R. E. 52 years - urethral discharge.

Patient with recurrent episodes of nonspecific urethritis with Staphylococcus aureus.

The culture was isolated S. aureus.

The antibiogram result:
S: AMC, SAM, CIP, LEV, RIF, VA, LZD
I: AM, AX
R: P., OX, ER., CLR., GE, NET, CLIND,

\section{CXM, CAZ, FEP, DAP}

\section{Case 2}

M.S. 24, Student

Cultures of acne skin lesions: Staphylococcus aureus

The antibiogram result:

\section{S: CIP, LEV, ENR, RIF, VA, LZD}

I DO, SAM

A: AM, AX AMC, P., OX., ER. CLR. GE, NET, CLIND, CXM, IF, FEP, DAP

\section{Case 3}

A. A. 32 years old, vaginitis

Cultures of vaginal discharge: S.aureus

The antibiogram result:

S: SAM, CIP, LEV, AZM., NET, RIF, VA, LZD

I: AM, AMC, AX, GE

FEP, DAP

R: P., OX, ER., CLR., CLIND, CXM, CAZ,

S: susceptible

I: intermediary

R: resistant

The combination of daptomycin and $\beta$-lactams may be useful for the treatment of MRSA infection, but further studies are needed to elucidate the mechanisms and to determine the in vivo efficacy of association.

In addition, testing indicated daptomycin dilution technique using MullerHinton broth liquid medium supplemented with $50 \mathrm{mg} / \mathrm{ml} \mathrm{Ca++}$, with the MIC determination.

Resistance to antibiotics reserve is an inevitable consequence of exposure to antibiotic selective pressure. Minimizing selection pressure favoring resistant strains is essential in controlling the emergence of such strains in both hospital and ambulatory. 
653-665

3. Davis, S.L., McKinnon, P.S. \& Hall, L.M. (2007). Daptomycin versus vancomycin for complicated skin and skin structure infections: clinical and

1. Daptomycin is very active in vitro to Gram - positive cocci, particularly methicillinresistant staphylococci, vancomycin-resistant enterococci and other penicillin-resistant streptococci.

2. We tested to daptomycin 112 strains of Gram-positive cocci: Staphylococcus aureus, Streptococcus agalactiae, Enterococcus spp. isolated in various pathological products.

3. Susceptibility to daptomycin of Staphylococcus aureus (95.89\%) was higher than susceptibility to vancomycin $(93.24 \%)$, but lower then sensitivity to teicoplanin $(97.29 \%)$.

4. In our study we did not identify Streptococcus agalactiae and Enterococcus spp resistant to daptomycin.

5. We found 5 strains of $\mathrm{S}$. aureus resistant to vancomycin sensitive to daptomycin, and 3 cases of daptomycin-resistant S. aureus and vancomycin-sensitive.

6. It can be appreciated that there were strains with multiple resistance: resistant to oxacillin (MRSA), macrolides, 2nd and 3rd generation of cephalosporins.

7. In the case of severe infections that require more aggressive treatment using antibiotics active on MRSA, Daptomycin is a valid therapeutic option, but the antibiogram is mandatory to ensure effective indication.

\section{References}

1. Daptomycin. (2006). In Klasco RK (Eds.), Drugdex system, vol. 129. Greenwood Village (CO): Thomson Micromedex

2. Fowler, V.G., Boucher, H.W. \& Corey, G.R. (2006). Daptomycin versus standard therapy for bacteremia and endocarditis caused by Staphylococcus aureus. $N$ Engl J Med, 355(7), 